\title{
Magnetic Resonance Imaging and Magnetic Resonance Spectroscopy for Detection of Early Alzheimer's Disease
}

\author{
Eric Westman ${ }^{\mathrm{b}, \mathrm{c}, *}$, Lars-Olof Wahlund ${ }^{\mathrm{a}}$, Catherine Foy ${ }^{\mathrm{b}}$, Michaela Poppe ${ }^{\mathrm{b}}$, Allison Cooper ${ }^{\mathrm{b}}$, \\ Declan Murphy ${ }^{\mathrm{b}}$, Christian Spenger ${ }^{\mathrm{d}}$, Simon Lovestone ${ }^{\mathrm{b}}$ and Andrew Simmons $\mathrm{s}, \mathrm{c}$ \\ ${ }^{a}$ Department of Neurobiology, Care Sciences and Society, Section of Clinical Geriatrics, Karolinska Institutet, \\ Karolinska University Hospital, Huddinge, Stockholm, Sweden \\ ${ }^{\mathrm{b}}$ NIHR Specialist Biomedical Research Centre for Mental Health at the South London and Maudsley \\ NHS Foundation Trust and King's College London and the MRC Centre for Neurodegeneration, \\ Kings College London, Institute of Psychiatry, London, UK \\ ${ }^{\mathrm{c}}$ Department of Neuroimaging, Kings College London, Institute of Psychiatry, London, UK \\ ${ }^{\mathrm{d}}$ Department of Clinical Science, Intervention and Technology, Karolinska Institutet, Stockholm, Sweden
}

\begin{abstract}
Alzheimer's disease is the most common form of neurodegenerative disorder and early detection is of great importance if new therapies are to be effectively administered. We have investigated whether the discrimination between early Alzheimer's disease (AD) and elderly healthy control subjects can be improved by adding magnetic resonance spectroscopy (MRS) measures to magnetic resonance imaging (MRI) measures.

In this study $30 \mathrm{AD}$ patients and 36 control subjects were included. High resolution T1-weighted axial magnetic resonance images were obtained from each subject. Automated regional volume segmentation and cortical thickness measures were determined for the images. ${ }^{1} \mathrm{H}$ MRS was acquired from the hippocampus and LCModel was used for metabolic quantification. Altogether, this yielded 58 different volumetric, cortical thickness and metabolite ratio variables which were used for multivariate analysis to distinguish between subjects with $\mathrm{AD}$ and Healthy controls. Combining MRI and MRS measures resulted in a sensitivity of $97 \%$ and a specificity of $94 \%$ compared to using MRI or MRS measures alone (sensitivity: $87 \%, 76 \%$, specificity: $86 \%, 83 \%$ respectively). Adding the MRS measures to the MRI measures more than doubled the positive likelihood ratio from 6 to 17 .

Adding MRS measures to a multivariate analysis of MRI measures resulted in significantly better classification than using MRI measures alone. The method shows strong potential for discriminating between Alzheimer's disease and controls.
\end{abstract}

Keywords: MRS, MRI, OPLS, AD, multivariate analysis

\section{INTRODUCTION}

Multivariate analysis provides the opportunity to analyze many variables simultaneously and observe

${ }^{*}$ Correspondence to: Eric Westman, PhD, Karolinska Universitetssjukhuset, Novum, Plan 4, 14186 Stockholm, Sweden. Tel.: ++4673 655 5179; Fax: ++468517 761 11. E-mail: eric.westman@ ki.se. inherent patterns in the data. Methods like principal component analysis (PCA), Partial least square to latent structures (PLS) and orthogonal PLS (OPLS) are efficient, robust and validated tools for modelling complex biological data [1].

Alzheimer's disease (AD) is one of the most common forms of neurodegenerative disorders. The clinical symptoms of AD include gradual loss of cognitive functions and $\mathrm{AD}$ is largely a disorder of the 
elderly with a small percentage of non-age-related $\mathrm{AD}$ cases being familial and secondary to specific gene mutations.

Magnetic resonance imaging (MRI) is a noninvasive method which has been widely studied for early detection and diagnosis of AD [2-4]. In particular early changes in hippocampus and entorhinal cortex have been demonstrated using MRI [5-9]. These early changes are consistent with the underlying pathology of $\mathrm{AD}$ but it is not yet clear which measures are most useful for early diagnosis [2]. Due to the complexity of this disorder measures of single structures from MRI are probably not sufficient for accurate diagnosis at the early stages of the disease. The most common way of describing the spread of atrophy in AD is according to the neurofibrillary tangle spread described by Braak and Braak [10]. It has also been proposed that the pattern of atrophy can progress in other ways, where the hippocampus and entorhinal cortex are not as affected [11]. By combining different measures of atrophy using multivariate methods we might gain a better understanding of the natural history of the disease.

Magnetic resonance spectroscopy $\left({ }^{1} \mathrm{H}-\mathrm{MRS}\right)$ provides useful information on the neurochemical profile of different neurodegenerative diseases $[12,13]$ from defined target volumes in vivo. The metabolites measured represent different aspects of the pathological processes in AD [13]. Examples of measurable metabolites are $\mathrm{N}$-acetylaspartate (NAA), a marker for neuronal density and/or function, myo-inositol (mI), a marker for astrogliosis and/or osmotic stress and choline (Cho), a marker for cell membrane turnover and degradation [14]. Brain metabolites are sensitive to pathological processes in neurodegenerative disorders such as AD [13].

Other MR modalities which can be used for the study of $\mathrm{AD}$ include diffusion MRI which measures microstructural changes in white matter [15], functional MRI which measures brain function using BOLD contrast [16], arterial spin labelling which measures the perfusion of blood [17] and MR-relaxometry which characterizes $T 1$ and $T 2$ relaxation times of tissue [18].

McEvoy et al. have previously shown with a largely automated image analysis pipeline that using multiple MRI measurements of regional volumes and regional cortical surface measurements in combination with multivariate analysis is useful in distinguishing between subjects with Alzheimer's disease and healthy controls [19]. This indicates that a combination of different MRI measures may prove to be more useful than hippocampal or entorhinal cortex measures alone for early detection of Alzheimer's disease. The use of automated measures may in particular have advantages when it comes to widespread uptake in either clinical or research practice. Several other studies have utilized different multivariate techniques including OPLS to analyze MR-data [20-26]. Alzheimer's disease is a complex disorder and one biomarker is probably not enough to establish a correct diagnosis. Therefore we wanted to investigate the potential of combining different MRI measures (i.e. regional volumes and regional cortical thickness measures) with MRS measures. OPLS was chosen to analyze the large number of variables generated from the different MR-modalities. The aim of this study was to investigate whether adding MRS measures to a battery of automated structural MRI measures would further improve the ability to distinguish patients with $\mathrm{AD}$ from healthy controls using multivariate data analysis.

\section{MATERIAL AND METHODS}

\section{Study data and inclusion and diagnostic criteria}

Thirty patients with Alzheimer's disease and 36 healthy volunteers were included in this study who had both MRI and hippocampal MRS. Table 1 gives the demographics of the study cohort. The study population was derived from a largely community-based population of subjects with $\mathrm{AD}$ and healthy elderly people [Alzheimer's Research Trust (ART) cohort] [27]. Community or nursing home resident cases with NINCDS-ADRDA (The National Institute of Neurological and Communicative Disorders and Stroke - the Alzheimer's disease and related Disorders Association) probable-AD were identified from secondary care

Table 1

Subject characteristics

\begin{tabular}{lcc}
\hline Variable & AD & CONTROL \\
\hline Number & 30 & 36 \\
Gender (female/male) & $15 / 15$ & $22 / 14$ \\
Mean age (SD) & $77,3(5,0)$ & $76,5(5,1)$ \\
Mean MMSE (SD) & $23,1(3,6)$ & $29,4(0,7)$ \\
Mean GDS (SD) & $4,0(0,7)$ & - \\
$\begin{array}{l}\text { Mean duration of disease } \\
\quad \text { years; SD) }\end{array}$ & $4,3(2,3)$ & - \\
$\begin{array}{l}\text { Mean years of education } \\
\quad(\text { years; SD) }\end{array}$ & $11,2(3,1)$ & $11,7(3,2)$ \\
\hline
\end{tabular}

Data are represented as average \pm standard deviation. $\mathrm{AD}=\mathrm{Alz}$ heimer's Disease, CONTROL $=$ healthy controls, $\mathrm{MMSE}=\mathrm{Mini}$ Mental State Examination and GDS = Global Dementia Scale . 
services for elderly people with dementia. In addition to a clinical diagnosis, subjects were assessed with a standardised assessment protocol including informant interview for diagnosis, MMSE and Global Dementia Scale (GDS) assessments for severity. Healthy volunteers were recruited from non-related members of the patient's families, caregiver's relatives or social centres for the elderly. The inclusion and exclusion criteria were as follows.

\section{Alzheimer's disease}

Inclusion criteria: (1) ADRDA/NINCDS and DSMIV criteria for probable Alzheimer's disease. (2) Mini Mental State Examination score range between 12 and 28. (3) Age 65 years or above. Exclusion criteria: (1) Significant neurological or psychiatric illness other than Alzheimer's disease. (2) Significant unstable systematic illness or organ failure.

\section{Controls}

Inclusion criteria: (1) Mini Mental State Examination score $>26$. (2) Geriatric Depression Scale score less than or equal to 5. (3) Age 65 years or above. (4) Medication stable. (5) Good general health. Exclusion criteria: (1) Meet the DSM-IV criteria for Dementia. (2) Significant neurological or psychiatric illness other than Alzheimer's disease. (3) Significant unstable systematic illness or organ failure.

Although additional subjects had MRI only the cohort considered here did not differ statistically from the larger cohort. MRI and MRS measures were not a part of the clinical evaluation and therefore did not influence the diagnostic decision. This study was approved by the South London and Maudsley NHS Trust research ethics committee.

\section{MRI and MRS acquisition}

Subjects were scanned using a 1.5 Tesla, GE NV/i Signa MR system (General Electric, Milwaukee, WI, USA) at the Maudsley Hospital, London. 3D T1weighted volume images were acquired in the axial plane with 1.5-mm contiguous sections using acquisition parameters chosen using a contrast simulation tool [28]. Repetition time (TR) was $13.8 \mathrm{~ms}$, inversion time (TI $450 \mathrm{~ms}$, echo time (TE) $2.8 \mathrm{~ms}$, and the flip angle was $20^{\circ}$ with one data average and a $256 \times 256 \times 124$ voxel matrix. Acquisition time was $6 \mathrm{~min}, 27 \mathrm{~s} .{ }^{1} \mathrm{H}-$
MRS voxels of interest measuring $20 \times 20 \times 15 \mathrm{~mm}^{3}$ $(6 \mathrm{~mL})$ were defined in standard locations in the left and right hippocampi using previously published methods [29]. We chose hippocampal regions of interest as this is one of the earliest sites of change in Alzheimer's disease. The anterior extent of the voxel was defined as the coronal slice where the amygdala disappeared, with the posterior extent $20 \mathrm{~mm}$ from this (Fig. 1A). The hippocampal volume of interest contained both grey and white matter and included the parahippocampal gyrus and the posterior portion of the amygdala. A point resolved spectroscopy (PRESS) pulse sequence (TE $35 \mathrm{~ms}$, TR $1500 \mathrm{~ms}, 256$ data averages and 2048 points) with automated shimming and water suppression and excellent reproducibility [30] was used to obtain spectra from each voxel after CHESS water suppression with high signal to noise ratio and clearly resolved NAA, Cho, $\mathrm{mI}$ and $\mathrm{Cr}+\mathrm{PCr}$ peaks among other metabolites. Not all subjects had spectral data from both left and right hippocampus. No significant differences were found in the metabolic content between the right and the left side of hippocampus. Therefore, we averaged the metabolic ratios from the left and right hippocampus from the subjects which had data from both hemispheres.

\section{MRI data analysis}

Freesurfer (version 5.0.0), a highly automated structural MRI image processing pipeline was utilised for data analysis. The pipeline produces regional cortical thickness and volumetric measures. Cortical reconstruction and volumetric segmentation includes removal of non-brain tissue using a hybrid watershed/surface deformation procedure [31], automated Talairach transformation, segmentation of the subcortical white matter and deep grey matter volumetric structures (including hippocampus, amygdala, caudate, putamen, ventricles) [31-33] intensity normalization [34], tessellation of the grey matter white matter boundary, automated topology correction [35, 36], and surface deformation following intensity gradients to optimally place the grey/white and grey/cerebrospinal fluid borders at the location where the greatest shift in intensity defines the transition to the other tissue class [37-39]. Once the cortical models are complete, registration to a spherical atlas takes place which utilizes individual cortical folding patterns to match cortical geometry across subjects [40]. This is followed by parcellation of the cerebral 

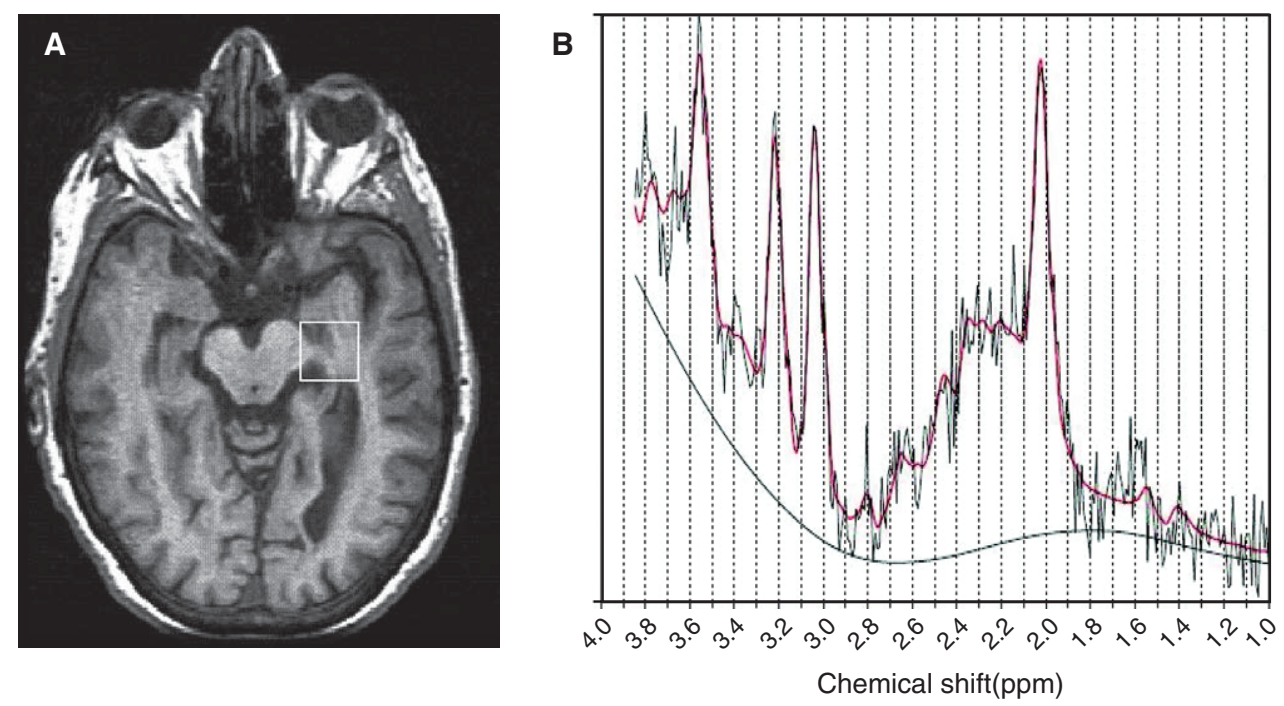

Fig. 1. (A) Representative axial T1-weighted magnetic resonance image of an AD patient illustrating the location of 1H-MRS voxel in the left hippocampus (B) Representative spectrum and model fit from LCModel output.

cortex into units based on gyral and sulcal structure $[41,42]$. Fig. 2A and B show representations of ROIs included as candidate input variables in the multivariate OPLS model. All volumetric measures from each subject were normalized by the subject's intracranial volume. This segmentation approach has previously been used for neuropsychological-image analysis [43, 44], imaging-genetic analysis [45-47] and biomarker discovery $[48,49]$.

\section{MRS data analysis}

The software package LCModel (http://www.sprovencher.com) $[50,51]$ was used for the analysis of the spectra. Fig. 1B shows a representative LCModel output, a spectrum with the model fit. The LCModel algorithm applies linear combinations of individual metabolite signals to calculate the best fit of the experimental spectra to the model spectra. In this case, a basis set of alanine, aspartate, creatine, gamma-aminobutyric acid (GABA), glutamine, glutamate, glycerophosphocholine, mI, lactate, NAA, $\mathrm{N}$-acetyl-aspartylglutamate (NAAg), scyllo-inositol, and taurine, together with a baseline function were used for analysis. As expected, many of the metabolite peaks included in the LC-model did not reach statistical significance when fitted; however those for
$\mathrm{NAA}, \mathrm{mI}, \mathrm{Cr}+\mathrm{PCr}$ and Cho did reach significant for all spectra. Metabolite concentration ratios relative to creatine + phosphocreatine $(\mathrm{Cr}+\mathrm{PCr})$ were calculated as applied by others [52, 53]. To ensure that differences in tissue composition did not account for metabolite differences between subject groups, we segmented the 3-D T1 weighted volume using SPM (Statistical Parametric Mapping) software (http:// www.fil.ion.bpmf.ac.uk/spm) to determine the percentage of grey and white matter and CSF composition within each MRS voxel. The metabolite concentrations reported by LCModel were divided by the fractional content of brain tissue $(\mathrm{p}[\mathrm{GM}]+\mathrm{p}[\mathrm{WM}]$, where $\mathrm{p}[\mathrm{GM}]$ and $\mathrm{p}[\mathrm{WM}]$ represent the percentage of grey matter and white matter in the voxel, respectively) to correct for the relative proportion of cerebrospinal fluid (CSF) in the MRS voxel (mean(SD) for $\mathrm{AD}=0.10(0.04)$ and for controls $=0.16(0.07), p=7.2 \mathrm{e}-6)$. The fraction $\mathrm{p}[\mathrm{GM}]$ was calculated for each subject to investigate if there were any significant differences in gray content between $\mathrm{AD}$ patients and control subjects in the MRS voxels. No significant differences were observed (mean(SD) for $\mathrm{AD}=0.62(0.07)$ and for controls $=0.65(0.06), p=0.60)$. The metabolite ratios included in the study were: $m y o$-inositol $(\mathrm{mI} / \mathrm{Cr}+\mathrm{PCr})$, choline-containing compounds $(\mathrm{Cho} / \mathrm{Cr}+\mathrm{PCr})$ and $\mathrm{N}$-acetylaspartate $+\mathrm{N}$-acetylaspartylglutamate (NAA $+\mathrm{NAAG} / \mathrm{Cr}+\mathrm{PCr}$ ). 

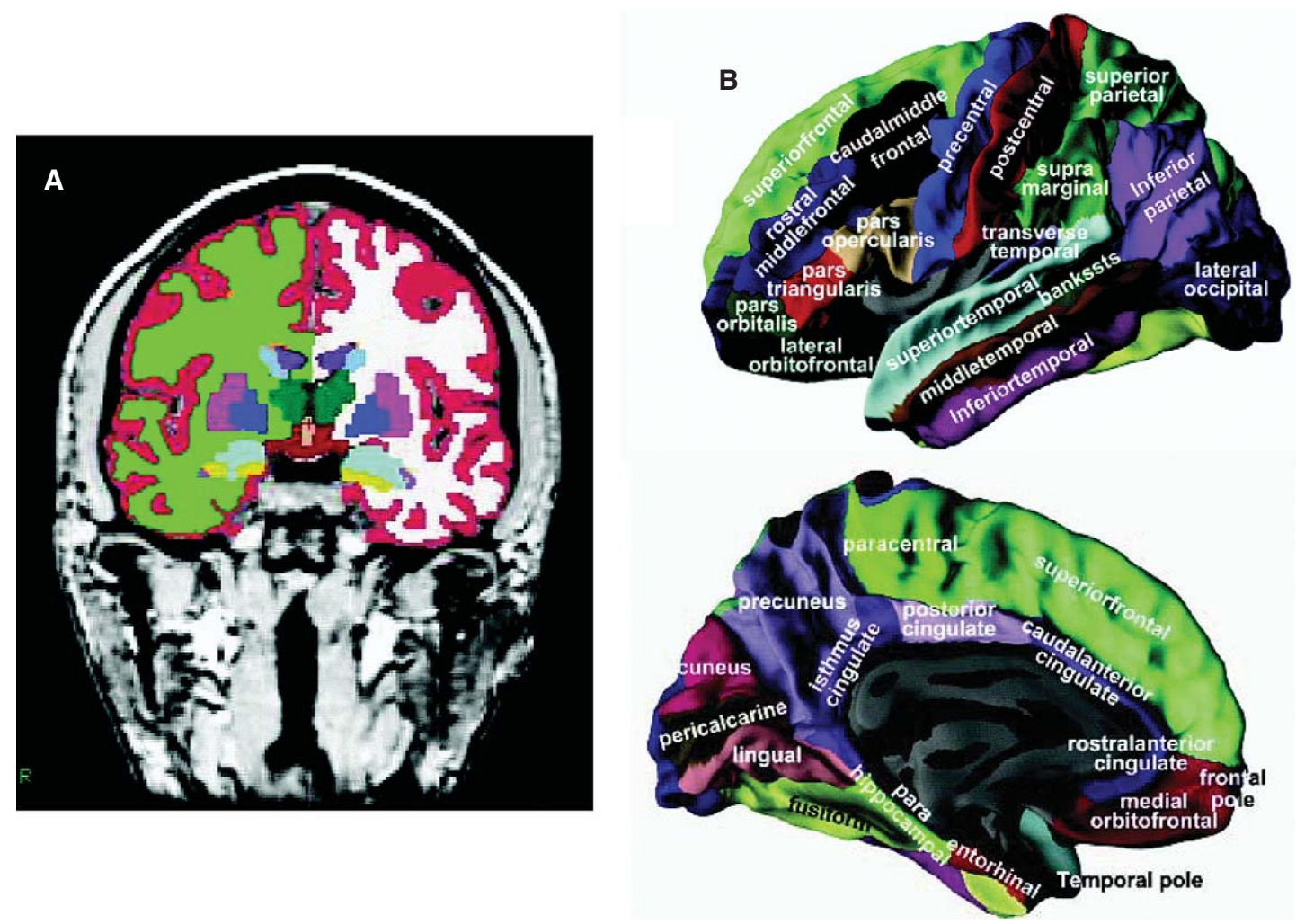

Fig. 2. Representations of ROIs included as candidate input variables in the multivariate OPLS model. (A) Regional volumes. (B) Regional cortical thickness measures.

\section{Multivariate data analysis}

MRI measures were analyzed using orthogonal partial least squares to latent structures (OPLS) [1, 24, 54-56], a supervised multivariate data analysis method included in the software package SIMCA (Umetrics $\mathrm{AB}$, Umea, Sweden). A very similar method, partial least squares to latent structures (PLS) has previously been used in several studies to analyze MR-data [23, $25,26,57,58]$. OPLS and PLS are very similar methods and when performed, give the same predictive accuracy. The advantage of OPLS compared to PLS is that the model created to compare groups is rotated. This means that the information related to class separation is found in the first component of the model, the predictive component. The other orthogonal components in the model, if any, relate to variation in the data not connected to class separation. Focusing the information related to class separation on the first component makes data interpretation easier [1].
Pre-processing was performed using mean centring and unit variance scaling. Mean centring improves the interpretability of the data, by subtracting the variable average from the data. By doing so the data set is repositioned around the origin. Large variance variables are more likely to be expressed in modeling than low variance variables. Consequently, unit variance scaling was selected to scale the data appropriately. This scaling method calculates the standard deviation of each variable. The inverse standard deviation is used as a scaling weight for each MR-measure.

The results from the OPLS analysis are visualized in a scatter plot by plotting the predictive component, which contains the information related to class separation. Components are vectors, which are linear combinations of partial vectors and are dominated by the input variables (x). The first and second components are by definition orthogonal to each other and span the projection plane of the points. Each point in the scatter plot represents one individual subject. 
The predictive component receives a $\mathrm{Q}^{2}(\mathrm{Y})$ value that describes its statistical significance for separating groups. $\mathrm{Q}^{2}(\mathrm{Y})$ values $>0.05$ are regarded as statistically significant and a model with a $\mathrm{Q}^{2}(\mathrm{Y})$ value $>0.5$ is regarded as good [59].

$$
\mathrm{Q}^{2}(\mathrm{Y})=1-\mathrm{PRESS} / \mathrm{SSY}
$$

PRESS (predictive residual sum of squares)= $\Sigma\left(\mathrm{y}_{\text {actual }}-\mathrm{y}_{\text {predicted }}\right)^{2}$ and SSY is the total variation of the Y matrix after scaling and mean centring [59]. $\mathrm{Q}^{2}(\mathrm{Y})$ is the fraction of the total variation of the $\mathrm{Ys}$ (expected class values) that can be predicted by a component according to cross validation (CV). Cross validation is a statistical method for validating a predictive model which involves building a number of parallel models. These models differ from each other by leaving out a part of the data set each time. The data omitted is then predicted by the respective model. In this study we used seven fold cross-validation, which means that $1 / 7$ th of the data is omitted for each crossvalidation round. Data is omitted once and only once. Variables were plotted according to their importance for the separation of groups. The plot shows the MRI measures and their corresponding jack-knifed confidence intervals. Jack-knifing is used to estimate the bias and standard error. Measures with confidence intervals that include zero have low reliability [1]. Covariance is plotted on the y-axis.

$$
\operatorname{Cov}(\mathrm{t}, \mathrm{Xi})=\mathrm{t}^{\mathrm{T}} \mathrm{X}_{\mathrm{i}} /(\mathrm{N}-1)
$$

Where $t$ is the transpose of the score vector $t$ in the OPLS model, $\mathrm{i}$ is the centered variable in the data matrix $\mathrm{X}$ and $\mathrm{N}$ is the number of variables [1]. A measure with high covariance is more likely to have an impact on group separation than a variable with low covariance. MRI and MRS measures below zero in the scatter plot have lower values in controls compared to $\mathrm{AD}$ subjects, while MRI and MRS measures above zero are higher in controls compared to AD subjects in the model.

Altogether 58 variables were used for OPLS analysis. No feature selection was performed, meaning all measured variables were included in the analysis. Three OPLS models were created. The first model contained MRS measures, the second model contained MRI measures and the third model combined both MRI and MRS measures. OPLS has previously been used to combine measures from different techniques $[54,56]$.

Models containing age, gender and education were also created to test if there were any significant differ- ences between the groups in these measures. Finally we also investigated whether age, gender and education would increase the predictive power of the models using them as $\mathrm{X}$-variables. As the models demonstrated no effect of age, gender and education these were excluded from further analysis.

The sensitivity and the specificity were calculated from the cross-validated prediction values received from the OPLS models. Finally, the positive likelihood ratios $(\mathrm{LR}+=$ sensitivity/(100-specificity)) were calculated. A positive likelihood ratio between 5-10 increases the diagnostic value in a moderate way, while a value above 10 significantly increases the diagnostic value of the test [60].

\section{RESULTS}

\section{Subject cohort}

Sixty-six subjects were included in this study: 30 $\mathrm{AD}$ patients and 36 controls as detailed in Table 1 . The gender distribution was equal for the AD subjects, but there were more females than males within the control group. Neuropsychological test results did not differ between females and males and gender differences were accounted for by dividing each regional volume by the subjects' intracranial volume. There were no significant differences between the two groups regarding age and education. As expected, the mean MMSE scores were significantly higher for the control group than the AD group. To measure the disease severity of the AD group the Global Dementia Scale was used. The mean value for the group was 4 , which corresponds to mild dementia. Finally the mean disease duration of the AD subjects was 4 years.

\section{OPLS modelling and quality}

Three models were created, the first using MRS measures, the second using MRI measures and the third model using both MRI and MRS measures. The first model (MRS measures) resulted in one predictive component. The model accounted for $59 \%$ of the variance of the original data $\left(\mathrm{R}^{2}(\mathrm{X})\right)$ and its' cross validated predictability, $\mathrm{Q}^{2}(\mathrm{Y})=31 \%$. The second model (MRI measures) resulted in one predictive component and one orthogonal component. For this model $\mathrm{R}^{2}(\mathrm{X})=59 \%$ and the cross validated predictability, $\mathrm{Q}^{2}(\mathrm{Y})=57 \%$. The third model (MRI + MRS) resulted in one predictive component and two orthogonal 
components. $\mathrm{R}^{2}(\mathrm{X})=62 \%$ and its' cross validated predictability, $\mathrm{Q}^{2}(\mathrm{Y})=67 \%$.

\section{Cross validated scatter plots}

Figure $3 \mathrm{~A}$ shows the separation between $\mathrm{AD}$ and controls using MRS measures. This resulted in a sensitivity of $76 \%$ and specificity of $83 \%$. In the model containing automated regional volume measures and regional cortical thickness measures a sensitivity of $87 \%$ and a specificity of $86 \%$ was found (Fig. 3B). The third model (MRI + MRS) had the highest prediction accuracy (Fig. 3C) yielding a sensitivity of $97 \%$ and a specificity of $94 \%$. The positive likelihood ratio more than doubled from 6 to 17 when the MRI and MRS measures were combined (Table 2). This significant improvement in diagnostic accuracy can also be observed in the increase of $\mathrm{Q}^{2}(\mathrm{Y})$ described above.
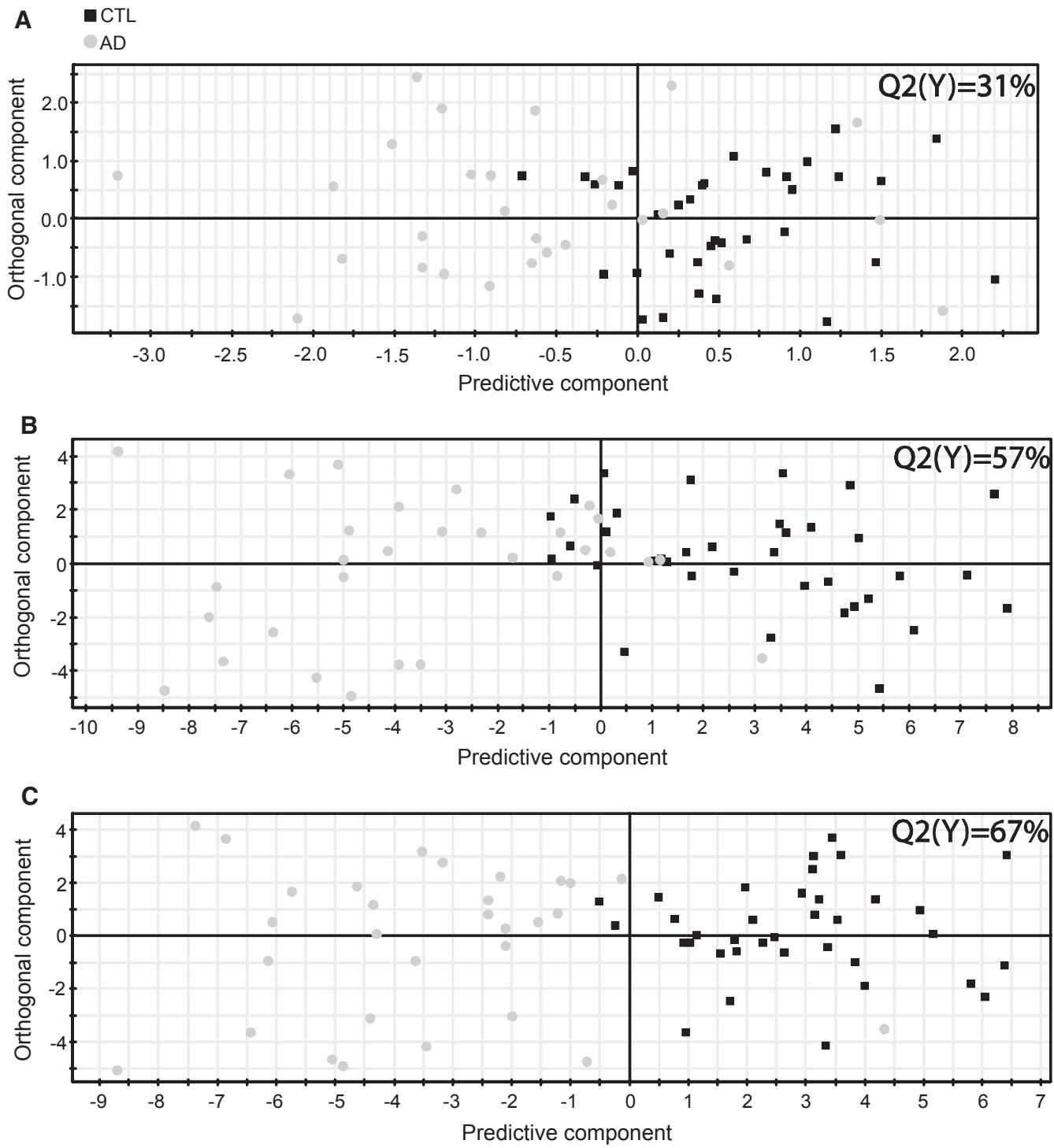

Fig. 3. OPLS cross validated score plots (A) MRS measures (B) MRI Measures (C) The combination of MRI and MRS measures. The scatter plots visualise group separation and the predictability of the three different AD vs. controls models. Each grey circle represents an AD subjects and each black square a control subject. Control subjects to the left of zero and AD subjects to the right of zero are falsely predicted. $\mathrm{Q}^{2}(\mathrm{Y})>0.05$ (statistically significant model) and $\mathrm{Q}^{2}(\mathrm{Y})>0.5($ good model $)$. 
Table 2

Sensitivity/specificity and positive Likelihood Ratio (LR+)

\begin{tabular}{lcccc}
\hline & \multicolumn{4}{c}{ AD vs. CONTROL } \\
\cline { 2 - 5 } & Sensitivity & Specificity & LR+ & $\mathrm{Q}^{2}(\mathrm{Y})$ \\
\hline MRS measures & $76 \%(59-88)$ & $83 \%(71-94)$ & $5(2-10)$ & 0.31 \\
MRI measures & $87 \%(70-95)$ & $86 \%(71-94)$ & $6(3-14)$ & 0.57 \\
MRI + MRS & & & \\
measures & $97 \%(83-99)$ & $94 \%(82-98)$ & $17(5-67)$ & 0.67 \\
\hline MRI = magnetic resonance imaging, MRS = magnetic & resonance \\
spectroscopy, AD = Alzheimer's disease, CONTROL = healthy con- \\
trols, LR+= likelihood ratio= sensitivity/(1-specificity), confidence \\
intervals for the sensitivity, specificity and LR+within parenthe- \\
sis, $\mathrm{Q}^{2}(\mathrm{Y})>0.05$ (statistically significant model), $\mathrm{Q}^{2}(\mathrm{Y})>0.5$ (good \\
model) and $\mathrm{Q}^{2}(\mathrm{Y})>0.7$ (excellent model).
\end{tabular}

\section{Variables responsible for separation}

Figure 4 illustrates the importance of the different variables in the model containing both MRI and MRS measures. Medial, lateral temporal lobe structures and isthmus cingulate as well as parietal and orbitofrontal regions were important for the separation between the two groups. The most important spectroscopic measure was $\mathrm{NAA} / \mathrm{Cr}+\mathrm{PCr}$.

\section{DISCUSSION}

Modern technology can allow high resolution MR images to be acquired in relatively short period of time which are suitable for making large numbers of mea- sures from. However it can be more challenging to study large numbers of patients due to cost and time constraints. Classical analysis methods such as multiple linear regression and analysis of variance assume statistical independence between variables and that the variables are highly relevant to the research question in hand [59]. The assumption that variables are statistically independent is not true when the number of variables exceeds the number of observations. Multivariate data analysis methods such as OPLS provide the opportunity to analyze many variables simultaneously. Unlike traditional methods, multivariate projection methods can also handle missing data and are robust to noise in both $\mathrm{X}$ and $\mathrm{Y}$ [59].

The OPLS method has previously been successfully applied by others to a wide range of data types $[1,54]$. Bylesjö et al. have shown that OPLS can be used to combine different types of omics data. They showed that the systematic variation from two analytical platforms could be combined and separated from the systematic variation specific to each analytical platform [54]. This illustrates one of the advantages of OPLS, that it divides the systematic variation within the data set into two parts, one correlated with $\mathrm{Y}$ and one uncorrelated with $\mathrm{Y}$, making data interpretation easier [1]. We have also recently used OPLS to analyze data from a large multi-center study (AddNeuroMed) using only structural MRI data as input variables [24].

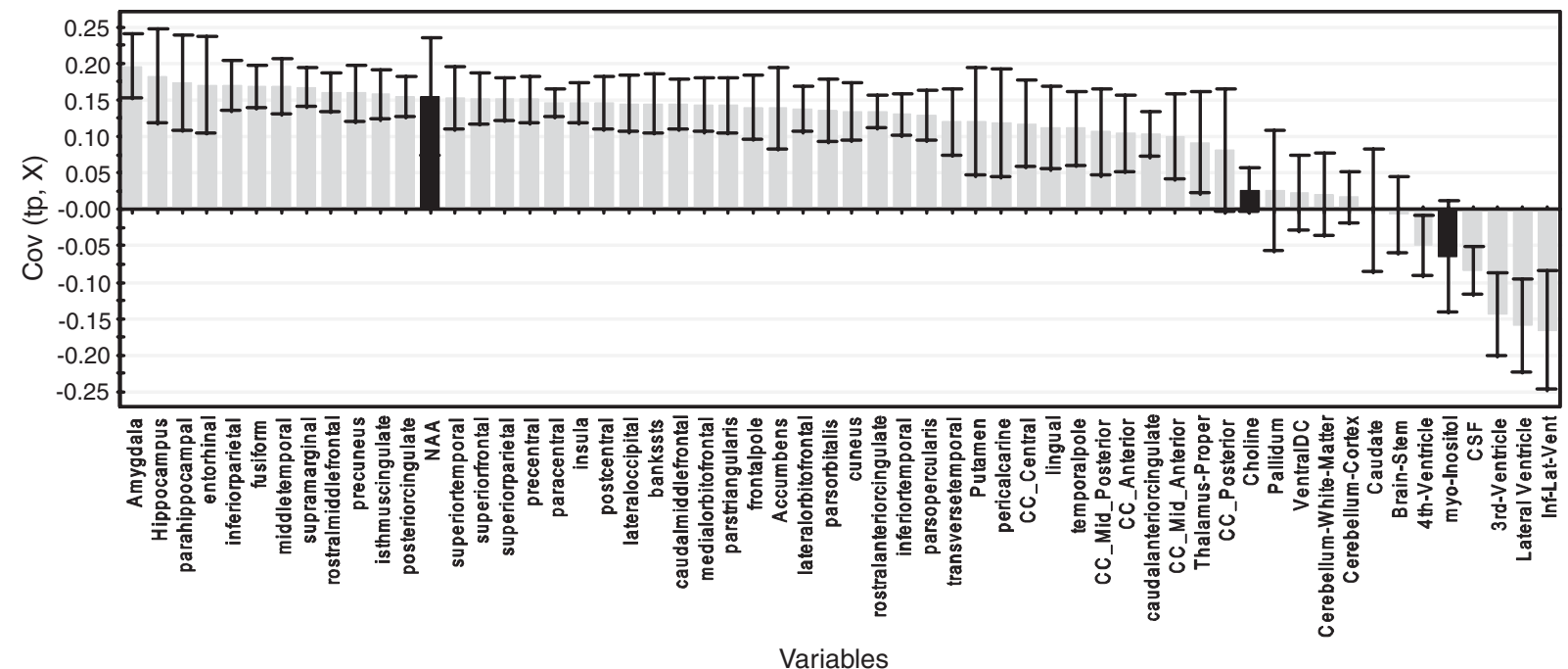

Fig. 4. MRI and MRS measures of importance for the separation between AD and controls. Measures above zero have a larger value in controls compared to $\mathrm{AD}$ and measures below zero have a lower value in controls compared to $\mathrm{AD}$. A measure with high covariance is more likely to have an impact on group separation than a measure with low covariance. Measures with jack knifed confidence intervals that include zero have low reliability. Measures in black are metabolite ratios and those in grey are regional volumes and cortical thickness measures. 


\section{Model predictability}

This study was designed to investigate the feasibility of discriminating between AD and controls using OPLS as a tool combining MRI measures with MRS measures. Several studies have used hippocampal or entorhinal cortex measures for classification between $\mathrm{AD}$ and controls with a high degree of accuracy $(80 \%-90 \%)[5-8,61-65]$. Other prior studies have shown up to $100 \%$ accuracy when discriminating between $\mathrm{AD}$ and controls but these studies either had very small sample size [66], included more severely impaired AD groups $[8,66,67]$ or did not use fully cross-validated results $[8,20,67,68]$. If crossvalidation is not used it can create an optimistic bias in classification accuracy [69, 70]. McEvoy et al. used linear discriminant analysis (LDA) on quantitative structural neuroimaging measures of regional MRI volumes and regional cortical thicknesses to distinguish between Alzheimer's disease and healthy controls [19]. They obtained a sensitivity of $83 \%$, a specificity of $93 \%$ and a positive likelihood ratio of 12 when comparing the two groups. By combining automated regional volumes and cortical thickness measures we found a sensitivity of $87 \%$ and specificity of $86 \%$ in the current study using the multivariate OPLS technique resulting in a positive likelihood ratio of 6 . We found a higher sensitivity but a lower specificity than McEvoy et al. for MRI measures alone which is reflected in the lower positive likelihood ratio. In another study Vemuri et al. used support vector machines (SVM) to classify subjects with probable AD from controls [71]. Including ApoE in their analysis they received a sensitivity of $88 \%$, a specificity of $90 \%$ resulting in a positive likelihood ratio of +9 . As before, we received a higher sensitivity and a lower specificity. Both the studies described above (Vemuri et al. and McEvoy et al.) are multi centre studies including larger cohorts of subjects, which may be the reason for the lower sensitivity values. Different centres can have slightly different inclusion criteria for AD patients, resulting in a more heterogeneous group. Several other studies have used SVM for discriminating between AD and controls with similar results to ours [21, 22, 72]. PLS, which is a similar method to OPLS, has also been utilized with success for the analysis of MR, PET and MEG data [23, 57].

We compared the discriminant ability of structural MRI analyses with those of MRS and to the combination of MRI and MRS. The model containing only MRS measures gave a sensitivity of $76 \%$ and speci- ficity of $83 \%(\mathrm{LR}+=5)$; somewhat less effective in discriminating $\mathrm{AD}$ from controls than the structural MRI analyses. The addition of MRS measures to the MRI measures improved the discrimination, however, resulting in a sensitivity of $97 \%$ and specificity of $94 \%$ which gave a positive likelihood ratio of 17 , better than that of McEvoy et al. The sensitivity and specificity of combined measures was greater than either MRI or MRS alone, with a more than doubling of the likelihood ratio. This significant improvement in diagnostic accuracy can also be observed in the increase of $\mathrm{Q}^{2}(\mathrm{Y})$ (Table 2). We have previously also used OPLS to distinguish between $\mathrm{AD}$ and controls using another automated pipeline combined with manual hippocampal volumes [24].

The OPLS multivariate method has previously been used to discriminate been groups successfully in other fields of research. Wiklund et al. used gas chromatography coupled mass spectroscopy data to differentiate between two transgenic poplar lines and wild type [1]. Another study combined data from two different platforms (2D-DIEGE proteomic and ${ }^{1} \mathrm{H}-\mathrm{NMR}$ metabolic data) to analyse blood plasma from mice with a prostate cancer xenograft and matched controls [56]. This study demonstrated that data from different analytical platforms can be successfully combined and gives us a better understanding of in vivo model systems.

\section{MR-measures of importance}

McEvoy et al. found that atrophy in medial and lateral temporal, isthmus cingulate and orbitofrontal areas aided the discrimination of control subjects from subjects with AD [19]. We also found that these regions were of importance for the cohort investigated in this study (Fig. 4). This demonstrates that the results can be reproduced and that the automated pipeline analysis methods used in both studies are robust. Decreased levels of NAA/Cr $+\mathrm{PCr}$ were observed in AD compared to controls and adding the spectroscopic measures increased the prediction accuracy. Kantarci et al. demonstrated that combining MRI with MRS improves the ability to identify patients with prodromal dementia [73]. Other studies have also shown that the combination of NAA and structural MRI improves the classification accuracy [74, 75]. This indicates the potential value of adding MR spectroscopic measures to volumetric measures in the diagnosis of $\mathrm{AD}$ and other neurodegerative disorders. Metabolic changes have previously been detected in presymptomatic mutation carriers years before expected onset 
[76] which demonstrates the potential use of MRS. It is possible that metabolite ratios provide less information about how individual metabolites change than metabolite concentrations. However, ratios are not susceptible to CSF partial volume effect and may represent more sensitive biomarkers of disease [77]. While we studied hippocampal MRS it would be interesting to apply the same technique to other regions of interest including the anterior cingulate using either single voxel MRS or magnetic resonance spectroscopic imaging (MRSI). A possible explanation for the added value of MRS to MRI is that they reflect different aspects of pathological processes, brain atrophy and metabolic changes. In $\mathrm{AD}$, molecular neuropathology is thought to precede structural brain changes by several years and the neurodegeneration is estimated to start 20-30 years before the clinical diagnosis is given [78]. These factors may play a role in explaining why these methods in combination improve the diagnostic outcome.

\section{Conclusion}

Quantitative MRI and MRS measurements in combination can improve the accuracy of discriminating patient with early Alzheimer's disease from normally cognitive elderly subjects over and above that of MRI measures and multivariate analysis alone. The multivariate method applied here (OPLS) provides the opportunity to analyze all of the MRI and MRS measures simultaneously, allowing the building of robust OPLS models for the prediction of disease demonstrating high sensitivity and specificity. Combining the automated MRS measures with MRI measures more than doubled the positive likelihood ratio from 6 to 17 which highlights the importance of MRS measures as a valuable complement to MRI in the diagnosis of Alzheimer's disease. A potential limitation of the current study is that the analysis technique was applied to a moderately sized MRI study with subjects recruited from a single site. Further, studies are warranted including those focusing on patients with mild cognitive impairment, patients with other types of dementia, longitudinal data and external test data sets to validate the robustness of the models. Applying this approach to typical clinical populations including mixed pathology and unclear clinical presentation would be of particular interest. Ultimately, pathologically confirmed data sets are needed to determine the applicability of the method described. However the improvement in discrimination when adding the MRS measures led to a clear and large improvement in the positive likelihood ratio. We chose a hippocampal region of interest for MRS since this is a particularly relevant area for AD and can be measured in a clinically realistic time. It would be of scientific interest in future to use the same approach described here for MR spectroscopic imaging or multiple single voxel MRS measures, though this may not be realistic for routine clinical imaging. To conclude, combining global and regional measures of atrophy with MRS measures significantly improves the classification accuracy when distinguishing between AD patients and healthy controls. Since all measures can be acquired with fully automated methods it makes them attractive for further use.

\section{ACKNOWLEDGEMENTS}

We acknowledge funding from the NIHR Biomedical Research Centre for Mental Health, the Alzheimer's Research Trust and the EU/EFPIA study, AddNeuroMed. Also thanks to the foundation Gamla Tjänarinnor, the foundation for Ragnhild och Einar Lundströms minne, The Swedish Alzheimer's Association, Swedish Brain Power and Stockholm Medical Image Laboratory and Education (SMILE). There are no actual or potential conflicts of interests.

\section{REFERENCES}

[1] Wiklund S, Johansson E, Sjostrom L, Mellerowicz EJ, Edlund U, Shockcor JP, Gottfries J, Moritz T, Trygg J (2008) Visualization of GC/TOF-MS-based metabolomics data for identification of biochemically interesting compounds using OPLS class models. Anal Chem 80, 115-122.

[2] O'Brien JT (2007) Role of imaging techniques in the diagnosis of dementia. Br J Radiol 80 Spec No 2, S71-S77.

[3] Ries ML, Carlsson CM, Rowley HA, Sager MA, Gleason CE, Asthana S, Johnson SC (2008) Magnetic resonance imaging characterization of brain structure and function in mild cognitive impairment: a review. J Am Geriatr Soc 56, 920-934.

[4] Scahill RI, Fox NC (2007) Longitudinal imaging in dementia. Br J Radiol 80 Spec No 2, S92-S98.

[5] Fox NC, Warrington EK, Freeborough PA, Hartikainen P, Kennedy AM, Stevens JM, Rossor MN (1996) Presymptomatic hippocampal atrophy in Alzheimer's disease. A longitudinal MRI study. Brain 119(Pt 6), 2001-2007.

[6] Jack CR Jr, Petersen RC, O'Brien PC, Tangalos EG (1992) MR-based hippocampal volumetry in the diagnosis of Alzheimer's disease. Neurology 42, 183-188.

[7] Jack CR Jr, Petersen RC, Xu YC, Waring SC, O'Brien PC, Tangalos EG, Smith GE, Ivnik RJ, Kokmen E (1997) Medial temporal atrophy on MRI in normal aging and very mild Alzheimer's disease. Neurology 49, 786-794.

[8] Juottonen K, Laakso MP, Partanen K, Soininen H (1999) Comparative MR analysis of the entorhinal cortex and 
hippocampus in diagnosing Alzheimer disease. AJNR Am J Neuroradiol 20, 139-144.

[9] Xu Y, Jack CR Jr, O’Brien PC, Kokmen E, Smith GE, Ivnik RJ, Boeve BF, Tangalos RG, Petersen RC (2000) Usefulness of MRI measures of entorhinal cortex versus hippocampus in AD. Neurology 54, 1760-1767.

[10] Braak H, Braak E (1991) Neuropathological stageing of Alzheimer-related changes. Acta Neuropathologica 82, 239259.

[11] Shiino A, Watanabe T, Maeda K, Kotani E, Akiguchi I, Matsuda M (2006) Four subgroups of Alzheimer's disease based on patterns of atrophy using VBM and a unique pattern for early onset disease. Neuroimage 33, 17-26

[12] Firbank MJ, Harrison RM, O'Brien JT (2002) A comprehensive review of proton magnetic resonance spectroscopy studies in dementia and Parkinson's disease. Dement Geriatr Cogn Disord 14, 64-76.

[13] Kantarci K (2007) $1 \mathrm{H}$ magnetic resonance spectroscopy in dementia. Br J Radiol 80 Spec No 2, S146-S152.

[14] Soares DP, Law M (2009) Magnetic resonance spectroscopy of the brain: review of metabolites and clinical applications. Clin Radiol 64, 12-21.

[15] Douaud G, Jbabdi S, Behrens TEJ, Menke RA, Gass A, Monsch AU, Rao A, Whitcher B, Kindlmann G, Matthews PM, Smith S (2011) DTI measures in crossing-fibre areas: Increased diffusion anisotropy reveals early white matter alteration in MCI and mild Alzheimer's disease. Neuroimage 55, 880-890.

[16] Sperling RA, Dickerson BC, Pihlajamaki M, Vannini P, LaViolette PS, Vitolo OV, Hedden T, Becker JA, Rentz DM, Selkoe DJ, Johnson KA (2010) Functional alterations in memory networks in early Alzheimer's disease. Neuromolecular Med 12, 27-43.

[17] Chen W, Song X, Beyea S, D’Arcy R, Zhang Y, Rockwood $\mathrm{K}$ (2011) Advances in perfusion magnetic resonance imaging in Alzheimer's disease. Alzheimer's and Dementia 7, 185-196.

[18] Deoni SC, Peters TM, Rutt BK (2005) High-resolution T1 and T2 mapping of the brain in a clinically acceptable time with DESPOT1 and DESPOT2. Magn Reson Med 53, 237-241.

[19] McEvoy LK, Fennema-Notestine C, Roddey JC, Hagler JDJ Jr, Holland D, Karow DS, Pung CJ, Brewer JB, Dale AM (2009) Alzheimer disease: Quantitative structural neuroimaging for detection and prediction of clinical and structural changes in mild cognitive impairment. Radiology 251, 195205.

[20] Fan Y, Batmanghelich N, Clark CM, Davatzikos C (2008) Spatial patterns of brain atrophy in MCI patients, identified via high-dimensional pattern classification, predict subsequent cognitive decline. Neuroimage 39, 1731-1743.

[21] Kloppel S, Stonnington CM, Barnes J, Chen F, Chu C, Good CD, Mader I, Mitchell LA, Patel AC, Roberts CC, Fox NC, Jack CR Jr, Ashburner J, Frackowiak RS (2008) Accuracy of dementia diagnosis: A direct comparison between radiologists and a computerized method. Brain 131, 2969-2974.

[22] Kloppel S, Stonnington CM, Chu C, Draganski B, Scahill RI, Rohrer JD, Fox NC, Jack CR Jr, Ashburner J, Frackowiak RS. (2008) Automatic classification of MR scans in Alzheimer's disease. Brain 131, 681-689.

[23] McIntosh AR, Lobaugh NJ (2004) Partial least squares analysis of neuroimaging data: Applications and advances. Neuroimage 23, S250-S263.
[24] Westman E, Simmons A, Zhang Y, Muehlboeck J, Tunnard C, Liu Y, Collins L, Evans A, Mecocci P, Vellas B, Tsolaki M, Kłoszewska I, Soininen H, Lovestone S, Spenger C, Wahlund L, consortium. ftA (2011) Multivariate analysis of MRI data for Alzheimer's disease, mild cognitive impairment and healthy controls. Neuroimage 54, 11781187.

[25] Westman E, Spenger C, Oberg J, Reyer H, Pahnke J, Wahlund LO (2009) In vivo $1 \mathrm{H}$-magnetic resonance spectroscopy can detect metabolic changes in APP/PS1 mice after donepezil treatment. BMC Neurosci 10,33.

[26] Westman E, Spenger C, Wahlund L-O, Lavebratt C (2007) Carbamazepine treatment recovered low $\mathrm{N}$ acetylaspartate $+\mathrm{N}$-acetylaspartylglutamate (tNAA) levels in the megencephaly mouse BALB/cByJ-Kv1.1 mceph/mceph. Neurobiology of Disease 26, 221-228.

[27] Hye A, Lynham S, Thambisetty M, Causevic M, Campbell J, Byers HL, Hooper C, Rijsdijk F, Tabrizi SJ, Banner S, Shaw CE, Foy C, Poppe M, Archer N, Hamilton G, Powell J, Brown RG, Sham P, Ward M, Lovestone S (2006) Proteome-based plasma biomarkers for Alzheimer's disease. Brain 129, 30423050 .

[28] Simmons A, Arridge SR, Barker GJ, Williams SCR (1996) Simulation of MRI cluster plots and application to neurological segmentation. Magnetic Resonance Imaging 14, 73-92.

[29] Robertson DMWM, van Amelsvoort TM, Daly EB, Simmons AP, Whitehead MM, Morris RGP, Murphy KCM, Murphy DGMM (2001) Effects of estrogen replacement therapy on human brain aging: An in vivo ${ }^{1} \mathrm{H}$ MRS study. Neurology 57 , 2114-2117.

[30] Simmons A, Smail M, Moore E, Williams SCR (1998) Serial precision of metabolite peak area ratios and water referenced metabolite peak areas in proton MR spectroscopy of the human brain. Magnetic Resonance Imaging 16, 319-330.

[31] Segonne F, Dale AM, Busa E, Glessner M, Salat D, Hahn HK, Fischl B (2004) A hybrid approach to the skull stripping problem in MRI. Neuroimage 22, 1060-1075.

[32] Fischl B, Salat DH, Busa E, Albert M, Dieterich M, Haselgrove C, van der Kouwe A, Killiany R, Kennedy D, Klaveness S, Montillo A, Makris N, Rosen B, Dale AM (2002) Whole brain segmentation: automated labeling of neuroanatomical structures in the human brain. Neuron 33, 341-355.

[33] Fischl B, Salat DH, van der Kouwe AJ, Makris N, Segonne F, Quinn BT, Dale AM (2004) Sequence-independent segmentation of magnetic resonance images. Neuroimage 23 Suppl 1, S69-S84.

[34] Sled JG, Zijdenbos AP, Evans AC (1998) A nonparametric method for automatic correction of intensity nonuniformity in MRI data. IEEE Trans Med Imaging 17, 87-97.

[35] Fischl B, Liu A, Dale AM (2001) Automated manifold surgery: constructing geometrically accurate and topologically correct models of the human cerebral cortex. IEEE Trans Med Imaging 20, 70-80.

[36] Segonne F, Pacheco J, Fischl B (2007) Geometrically accurate topology-correction of cortical surfaces using nonseparating loops. IEEE Trans Med Imaging 26, 518-529.

[37] Dale AM, Fischl B, Sereno MI (1999) Cortical surface-based analysis. I. Segmentation and surface reconstruction. Neuroimage $\mathbf{9}, 179-194$.

[38] Dale AM, Sereno MI (1993) Improved localizadon of cortical activity by combining EEG and MEG with MRI cortical sur- 
face reconstruction: A linear approach. Journal of Cognitive Neuroscience 5, 162-176.

[39] Fischl B, Dale AM (2000) Measuring the thickness of the human cerebral cortex from magnetic resonance images. Proc Natl Acad Sci U S A 97, 11050-11055.

[40] Fischl B, Sereno MI, Tootell RB, Dale AM (1999) Highresolution intersubject averaging and a coordinate system for the cortical surface. Hum Brain Mapp 8, 272-284.

[41] Desikan RS, Ségonne F, Fischl B, Quinn BT, Dickerson BC, Blacker D, Buckner RL, Dale AM, Maguire RP, Hyman BT, Albert MS, Killiany RJ (2006) An automated labeling system for subdividing the human cerebral cortex on MRI scans into gyral based regions of interest. NeuroImage 31, 968-980.

[42] Fischl B, van der Kouwe A, Destrieux C, Halgren E, Segonne F, Salat DH, Busa E, Seidman LJ, Goldstein J, Kennedy D, Caviness V, Makris N, Rosen B, Dale AM (2004) Automatically parcellating the human cerebral cortex. Cereb Cortex 14, 11-22.

[43] Liu Y, Paajanen T, Zhang Y, Westman E, Wahlund L-O, Simmons A, Tunnard C, Sobow T, Mecocci P, Tsolaki M, Vellas B, Muehlboeck S, Evans A, Spenger C, Lovestone S, Soininen H (2009) Combination analysis of neuropsychological tests and structural MRI measures in differentiating AD, MCI and control groups-The AddNeuroMed study. Neurobiol Aging. In press, Corrected Proof.

[44] Liu Y, Paajanen T, Zhang Y, Westman E, Wahlund L-O, Simmons A, Tunnard C, Sobow T, Mecocci P, Tsolaki M, Vellas B, Muehlboeck S, Evans A, Spenger C, Lovestone S, Soininen H (2010) Analysis of regional MRI volumes and thicknesses as predictors of conversion from mild cognitive impairment to Alzheimer's disease. Neurobiology of Aging 31, 1375-1385.

[45] Furney SJ, Simmons A, Breen G, Pedroso I, Lunnon K, Proitsi P, Hodges A, Powell J, Wahlund LO, Kloszewska I, Mecocci P, Soininen H, Tsolaki M, Vellas B, Spenger C, Lathrop M, Shen L, Kim S, Saykin AJ, Weiner MW, Lovestone S (2010) Genome-wide association with MRI atrophy measures as a quantitative trait locus for Alzheimer's disease. Mol Psychiatry 2010 Nov 30. [Epub ahead of print]

[46] Liu Y, Paajanen T, Westman E, Wahlund LO, Simmons A, Tunnard C, Sobow T, Proitsi P, Powell J, Mecocci P, Tsolaki M, Vellas B, Muehlboeck S, Evans A, Spenger C, Lovestone S, Soininen H (2010) Effect of APOE epsilon4 allele on cortical thicknesses and volumes: the Add Neuro Med study. $J$ Alzheimers Dis 21, 947-966.

[47] Liu Y, Paajanen T, Westman E, Zhang Y, Wahlund LO, Simmons A, Tunnard C, Sobow T, Proitsi P, Powell J, Mecocci P, Tsolaki M, Vellas B, Muehlboeck S, Evans A, Spenger C, Lovestone S, Soininen H (2010) APOE epsilon2 allele is associated with larger regional cortical thicknesses and volumes. Dement Geriatr Cogn Disord 30, 229-237.

[48] Thambisetty M, Hye A, Foy C, Daly E, Glover A, Cooper A, Simmons A, Murphy D, Lovestone S (2008) Proteome-based identification of plasma proteins associated with hippocampal metabolism in early Alzheimer's disease. J Neurol 255, 17121720 .

[49] Thambisetty M, Simmons A, Velayudhan L, Hye A, Campbell J, Zhang Y, Wahlund LO, Westman E, Kinsey A, Guntert A, Proitsi P, Powell J, Causevic M, Killick R, Lunnon K, Lynham S, Broadstock M, Choudhry F, Howlett DR, Williams RJ, Sharp SI, Mitchelmore C, Tunnard C, Leung R, Foy C, O'Brien D, Breen G, Furney SJ, Ward M, Kloszewska I, Mecocci P, Soininen H, Tsolaki M, Vellas B, Hodges A, Murphy DG, Parkins S, Richardson JC, Resnick SM, Ferrucci L, Wong DF, Zhou Y, Muehlboeck S, Evans A,
Francis PT, Spenger C, Lovestone S (2010) Association of plasma clusterin concentration with severity, pathology, and progression in Alzheimer disease. Arch Gen Psychiatry 67, 739-748.

[50] Provencher SW (1993) Estimation of metabolite concentrations from localized in vivo proton NMR spectra. Magn Reson Med 30, 672-679.

[51] Provencher SW (2001) Automatic quantitation of localized in vivo ${ }^{1} \mathrm{H}$ spectra with LCModel. NMR Biomed 14, 260-264.

[52] Ackl N, Ising M, Schreiber YA, Atiya M, Sonntag A, Auer DP (2005) Hippocampal metabolic abnormalities in mild cognitive impairment and Alzheimer's disease. Neurosci Lett 384, 23-28.

[53] Westman E, Spenger C, Wahlund LO, Lavebratt C (2007) Carbamazepine treatment recovered low $\mathrm{N}$-acetylaspartate $+\mathrm{N}$ acetylaspartylglutamate (tNAA) levels in the megencephaly mouse BALB/cByJ-Kv1.1(mceph/mceph). Neurobiol Dis 26, 221-228.

[54] Bylesjo M, Eriksson D, Kusano M, Moritz T, Trygg J (2007) Data integration in plant biology: the O2PLS method for combined modeling of transcript and metabolite data. Plant J 52, 1181-1191.

[55] Johan Trygg SW (2002) Orthogonal projections to latent structures (O-PLS). Journal of Chemometrics 16, 119-128.

[56] Rantalainen M, Cloarec O, Beckonert O, Wilson ID, Jackson D, Tonge R, Rowlinson R, Rayner S, Nickson J, Wilkinson RW, Mills JD, Trygg J, Nicholson JK, Holmes E (2006) Statistically integrated metabonomic-proteomic studies on a human prostate cancer xenograft model in mice. J Proteome Res $\mathbf{5}$, 2642-2655.

[57] Levine B, Kovacevic N, Nica EI, Cheung G, Gao F, Schwartz ML, Black SE (2008) The Toronto traumatic brain injury study: Injury severity and quantified MRI. Neurology 70, 771-778.

[58] Oberg J, Spenger C, Wang FH, Andersson A, Westman E, Skoglund P, Sunnemark D, Norinder U, Klason T, Wahlund LO, Lindberg M (2008) Age related changes in brain metabolites observed by (1)H MRS in APP/PS1 mice. Neurobiol Aging 29, 1423-1433.

[59] Eriksson L, Johansson E, Kettaneh-Wold N, Trygg J, Wiksröm C, Wold S (2006) Multi- and Megavariate Data Analysis (Part I -Basics and Principals and Applications), Umetrics AB, Umeå.

[60] Qizilbash S, Chui, Tariot, Brodaty, Kaye, Erkinjuntti (2002) (Blackwell Publishing, Oxford), 20-23.

[61] Killiany RJ, Moss MB, Albert MS, Sandor T, Tieman J, Jolesz F (1993) Temporal lobe regions on magnetic resonance imaging identify patients with early Alzheimer's disease. Arch Neurol 50, 949-954.

[62] Laakso MP, Partanen K, Riekkinen P, Lehtovirta M, Helkala EL, Hallikainen M, Hanninen T, Vainio P, Soininen H (1996) Hippocampal volumes in Alzheimer's disease, Parkinson's disease with and without dementia, and in vascular dementia: An MRI study. Neurology 46, 678-681.

[63] Laakso MP, Soininen H, Partanen K, Lehtovirta M, Hallikainen M, Hanninen T, Helkala EL, Vainio P, Riekkinen PJ Sr (1998) MRI of the hippocampus in Alzheimer's disease: sensitivity, specificity, and analysis of the incorrectly classified subjects. Neurobiol Aging 19, 23-31.

[64] Lehericy S, Baulac M, Chiras J, Pierot L, Martin N, Pillon B, Deweer B, Dubois B, Marsault C (1994) Amygdalohippocampal MR volume measurements in the early stages of Alzheimer disease. AJNR Am J Neuroradiol 15, 929937. 
[65] Seab JP, Jagust WJ, Wong ST, Roos MS, Reed BR, Budinger TF (1988) Quantitative NMR measurements of hippocampal atrophy in Alzheimer's disease. Magn Reson Med 8, 200208.

[66] Lerch JP, Pruessner J, Zijdenbos AP, Collins DL, Teipel SJ, Hampel H, Evans AC (2008) Automated cortical thickness measurements from MRI can accurately separate Alzheimer's patients from normal elderly controls. Neurobiol Aging 29, 23-30.

[67] Callen DJ, Black SE, Gao F, Caldwell CB, Szalai JP (2001) Beyond the hippocampus: MRI volumetry confirms widespread limbic atrophy in AD. Neurology 57, 1669-1674.

[68] Killiany RJ, Hyman BT, Gomez-Isla T, Moss MB, Kikinis R, Jolesz F, Tanzi R, Jones K, Albert MS (2002) MRI measures of entorhinal cortex vs hippocampus in preclinical AD. Neurology 58, 1188-1196.

[69] Schulerud H, Albregtsen F (2004) Many are called, but few are chosen. Feature selection and error estimation in high dimensional spaces. Comput Methods Programs Biomed 73, 91-99.

[70] Simon Spycher MNJG (2004) Comparison of different classification methods applied to a mode of toxic action data set. OSAR \& Combinatorial Science 23, 779-791.

[71] Vemuri P, Gunter JL, Senjem ML, Whitwell JL, Kantarci K, Knopman DS, Boeve BF, Petersen RC, Jack CR Jr (2008) Alzheimer's disease diagnosis in individual subjects using structural MR images: validation studies. Neuroimage $\mathbf{3 9}$, 1186-1197.

[72] Plant C, Teipel SJ, Oswald A, Böhm C, Meindl T, MouraoMiranda J, Bokde AW, Hampel H, Ewers M (2009) Automated detection of brain atrophy patterns based on MRI for the prediction of Alzheimer's disease. Neuroimage $\mathbf{5 0}$ 162-174.
[73] Kantarci K, Weigand SD, Przybelski SA, Shiung MM, Whitwell JL, Negash S, Knopman DS, Boeve BF, O'Brien PC, Petersen RC, Jack CR Jr (2009) Risk of dementia in MCI: Combined effect of cerebrovascular disease, volumetric MRI, and $1 \mathrm{H}$ MRS. Neurology 72, 1519-1525.

[74] Jessen F, Traeber F, Freymann N, Maier W, Schild HH, Heun R, Block W (2005) A Comparative Study of the Different N-Acetylaspartate Measures of the Medial Temporal Lobe in Alzheimerâ $€^{\mathrm{TM}_{\mathrm{S}}}$ Disease. Dementia and Geriatric Cognitive Disorders 20, 178-183.

[75] Schuff N, Capizzano AA, Du AT, Amend DL, O’Neill J, Norman D, Kramer J, Jagust W, Miller B, Wolkowitz OM, Yaffe K, Weiner MW (2002) Selective reduction of Nacetylaspartate in medial temporal and parietal lobes in AD. Neurology 58, 928-935.

[76] Godbolt AK, Waldman AD, MacManus DG, Schott JM, Frost C, Cipolotti L, Fox NC, Rossor MN (2006) MRS shows abnormalities before symptoms in familial Alzheimer disease. Neurology 66, 718-722.

[77] Bartha R, Smith M, Rupsingh R, Rylett J, Wells JL, Borrie MJ (2008) High field (1)H MRS of the hippocampus after donepezil treatment in Alzheimer disease. Prog Neuropsychopharmacol Biol Psychiatry 32, 786-793.

[78] Blennow K, de Leon MJ, Zetterberg H (2006) Alzheimer's disease. The Lancet 368, 387-403. 